\title{
Gebäudesanierung zwischen Energieeffizienz und Sozialverträglichkeit
} Zwei empirische Fallstudien

Sonja Haug, Institut für Sozialforschung und Technikfolgenabschätzung, Ostbayerische Technische Hochschule Regensburg, Seybothstr. 2, 93053 Regensburg (sonja.haug@oth-regensburg.de)

Miriam Vetter, Institut für Sozialforschung und Technikfolgenabschätzung, Ostbayerische Technische Hochschule Regensburg (miriam.vetter@oth-regensburg.de) Karsten Weber, Institut für Sozialforschung und Technikfolgenabschätzung, Ostbayerische Technische Hochschule Regensburg (karsten.weber@oth-regensburg.de)

Die Akzeptanz unter Bewohnern und Bewohnerinnen gewinnt bei hoch komplexen, technisch anspruchsvollen energetischen Sanierungen als "Innovationsmotor" zunehmend an Relevanz. Der Beitrag basiert auf zwei Fallstudien zur partizipativen Nutzereinbindung bei energetischen Sanierungen im genossenschaftlichen Wohnbau historischer Stadtquartiere in Regensburg. Neben einer sozialverträglichen Sanierung wurde jeweils ein hohes Maß an Energieeffizienz bei den technischen Lösungen angestrebt. Haushaltsbefragungen und qualitative Interviews zeigen die hohe Akzeptanz von Sanierungsmaßnahmen, sofern die Senkung der Energiekosten die erhöhte Miete kompensiert. Abschließend werden Akzeptanzfaktoren wie Partizipation, Vertrauen, Sozialverträglichkeit und Autarkie erörtert.

\section{Building restoration between energy efficiency and user acceptance Two empirical case studies}

Acceptance among residents is becoming increasingly important in establishing highly complex technical energy systems and energy-efficient modernization measures. This paper is based on two case studies on user involvement in the energy-efficient restoration of two cooperative owned building complexes in historic quarters of Regensburg/ Germany. Household surveys and qualitative interviews show the high acceptance of restoration measures, provided that the reduction of energy costs compensates for the increase in rents. Finally, acceptance factors such as participation, trust, social acceptability, and self-sufficiency are discussed.

Keywords: energy-efficient building restoration, user acceptance, household survey, historic quarter, renewable energy

This is an article distributed under the terms of the Creative Commons Attribution License CCBY 4.0 (https://creativecommons.org/licenses/by/4.0/)

https://doi.org/10.14512/tatup.29.3.56

Submitted: 17. 04.2020. Peer reviewed. Accepted: 22.07.2020

\section{Einleitung}

Der vorliegende Beitrag untersucht Akzeptanz von technischen Innovationen bei der Gebäudesanierung als einen wesentlichen sozialen Aspekt für den Erfolg der Energiewende (Wüstenhagen et al. 2007, S. 2684). Gebäudesanierungen können die Lebensqualität insbesondere von Personen im Mietverhältnis verbessern und die Energieeffizienz von Gebäuden erhöhen. Allerdings stagniert die nationale Sanierungsquote auf niedrigem Niveau bei etwa einem Prozent pro Jahr (Deutsche EnergieAgentur 2018, S. 15), obwohl die Bundesregierung verstärkt die Sanierung von Wohnungsbeständen fordert, um nationale Klimaziele einzuhalten (Weiß et al. 2012, S. 14).

Unsere sozialwissenschaftliche Begleitforschung untersucht die Akzeptanz technischer Innovationen und Geschäftsmodelle zur sozialverträglichen Gebäudesanierung bei zwei energetischen Sanierungsprojekten bei Wohnungsbaugenossenschaften im Regensburger Stadtgebiet. Hier wurden die Haushalte vor Beginn der Baumaßnahmen mit einem Fragebogen über ihre Akzeptanz zum Sanierungsvorhaben und Bedarfe bei baulichen Veränderungen im Quartier jeweils schriftlich befragt (ex-anteForschung). Neben der standardisierten Querschnittsbefragung wurde bei einer Fallstudie auch eine qualitative Interviewstudie durchgeführt. Die Bewohnerwünsche wurden erhoben, um sie bei der Planung mit zu berücksichtigen. Eine Umsetzung der Schlussfolgerungen im Praxisforschungsbericht und Leitfaden (Steffens et al. 2016) fand während der Projektlaufzeit nicht statt. Bei der Fallstudie MAGGIE erfolgte die Erprobung während des Projekts in einem Testgebäude. Neuartig ist die technische Entwicklung mit folgender Baumaßnahme und sozialwissenschaftlicher Begleitforschung. Erstmalig geht der Beitrag auf soziale Aspekte dieses Fallbeispiels ein und zeigt Ergebnisse eines Reliabilitätstests durch eine wiederholte Akzeptanzbefragung. 


\section{Theoretischer Hintergrund}

\section{Bewohnereinbindung in die Planung einer Sanierungsmaßnahme}

Nach Tappeiner et al. (2004, S. 141) kann das Expertenwissen der Bewohnerschaft bei einer Bewohnereinbindung einen Beitrag zu einem innovativen Sanierungskonzept leisten und so als "Innovationsmotor" wirken. Insbesondere die Akzeptanz der energetischen Sanierung konnte durch Einbindung der Bewohnerschaft gesteigert werden (Hacke und Lohmann 2006, S. 35 , S. 86).

Neben Besichtigungen, Veröffentlichungen und Informationsveranstaltungen kommt Bürgerbefragungen eine sehr wichtige Bedeutung als Beteiligungsform zu. Unten soll gezeigt werden, wie eine Berücksichtigung von Befragungsergebnissen bei der Planung von Sanierungsmaßnahmen mit erneuerbaren Energien möglich ist. Bei Genossenschaften kommt noch ein Mitspracherecht bei Entscheidungen über die Sanierung hinzu (siehe folgenden Abschnitt).

Die Bewohnereinbindung impliziert jedoch nicht automatisch eine hohe Sanierungsbereitschaft. Aufseiten der Bewohnerschaft sind Alter, Familienstand oder Haushaltseinkommen (Tappeiner et al. 2004, S. 141) ebenso Einflussfaktoren wie aufseiten der Hauseigentümerinnen und -eigentümer ökonomische Aspekte oder fehlendes technisches Fachwissen (Koch und Rosenberger 2019, S. 20). Letzteres kann z. B. durch unabhängige Energieberatungsstellen adressiert werden, stellt aber auch arbeitsorganisatorische Herausforderungen für den fortlaufenden Betrieb energieeffizienter Gebäudetechnik (Böhm et al. 2019).

\section{Rolle von Wohnungsbaugenossenschaften bei Gebäudemodernisierungen}

Eine energetische Gebäudesanierung kann als Investition in das Kollektivgut Weltklima angesehen werden, unterliegt somit aber auch dem Kollektivgutproblem: Kollektive Interessen werden oft nicht realisiert, weil es rational ist, auf das Engagement anderer zu warten (Diekmann und Preisendörfer 2001, S. 75). Faktoren, die die Bewirtschaftung eines Gemeinguts unter diesen tiersentwicklungen“ auf (Heinze und Bölting 2019, S. 65), z. B. sind sie aus Perspektive der Kommunen ein Erfolgsfaktor bei der Nutzung erneuerbarer Energien (Wehnert et al. 2007, S. 5). Da das anteilige Eigentum stärker zu einer Modernisierung und Werterhaltung des Baubestandes motiviert, müsste demnach bei Wohnungsbaugenossenschaften im Vergleich zu anderen Akteuren eine höhere Bereitschaft zu Klimaschutzmaßnahmen, Steigerung der Energieeffizienz von Gebäuden und energetischen Sanierungen vorliegen. Da Vorstand und Mitglieder demokratisch entscheiden, ist auch mit weniger Konflikten als beispielsweise bei Mietern und privaten Wohnungsunternehmen zu rechnen (Atmaca 2014, S. 66). Zudem beteiligen sich Baugenossenschaften aktiv an der Gestaltung des lokalen Umfelds und vertreten auch sozial benachteiligte Bewohnergruppen wie ältere Menschen (BMVBW 2004, S. 383).

\section{Anreizstrukturen und Motive für die Gebäudesanierung}

Eine energetische Gebäudesanierung trägt durch die Reduktion des $\mathrm{CO}_{2}$-Ausstoßes zum Kollektivgut Weltklima bei. Doch Hauseigentümerinnen oder Mieter haben in der Handlungsstruktur eines Kollektivgutproblems wenig Motivation, Mehrkosten aufzuwenden, selbst wenn sie eine Präferenz für Umweltschutz haben und die energetische Sanierung hohe Akzeptanzwerte hat. Daher können finanzielle Anreize zur Förderung der energetischen Sanierung von Wohngebäuden oder zur Einspeisung regenerativ erzeugter Energie Sanierungsmaßnahmen (mit) auslösen (Haug et al. 2017, S. 587). Um Energieziele einzuhalten, bieten bspw. die Energieeinsparverordnung (EnEV), Förderprogramme der Kreditanstalt für Wiederaufbau (KfW) oder das Erneuerbare-Energien-Wärmegesetz finanzielle Anreize (Vögele et al. 2016, S. 1).

Wilson et al. (2015, S. 15) analysierten Kosteneinsparungen, Komfort und Nutzen für die Umwelt als Motive sowie Sanierungskosten und Informationsdefizite als Hemmnisse bei der energieeffizienten Sanierung. Weiterhin gibt es persönliche Einflüsse wie Information und Bewusstsein, Einstellungen und Meinungen, Erfahrungen und Kenntnisse sowie kontextuelle Einflüsse wie Merkmale des Haushalts, soziostrukturelle Merk-

\section{Finanzielle Anreize können die Akzeptanz energetischer Sanierungen erhöhen.}

Bedingungen erfolgreich werden lassen, sind Restriktion des Zugangs zum Kollektivgut, Umweltangepasstheit, Partizipation, Monitoring, Sanktionierbarkeit, Konfliktregulierung und Autonomie (Diekmann und Preisendörfer 2001, S. 92-93).

Wie Ostrom (1999) zeigt, sind Genossenschaften für die Lösung einer Kollektivgutproblematik besonders geeignete Institutionen. Ebenso weisen Genossenschaften durch ihre Organisationsform auch ein hohes Potenzial für ,,zukunftsfähige Quar- male, Wohnsitz, Charakteristik des Gebäudes sowie Anreize durch die Politik. Eine qualitative Erhebung der Sanierungsmotive ergab, dass Wirtschaftlichkeit, Kosteneinsparungen, die Erhöhung des Wohnkomforts, z. B. durch Behebung von Störfaktoren, für Gebäudeeigentümer sehr bedeutsam sind. Des Weiteren wurde auch die Nutzung regenerativer Energie genannt (Gossen und Nischan 2014, S. 34). Für Wohnbaugenossenschaften bietet sich auch die Möglichkeit, Mitglieder und Mieter für Be- 
teiligung an Genossenschaftsstromerzeugung durch Anreize wie Stromkostenvorteile oder Umweltaspekte zu gewinnen (Kamburow et al. 2016, S. 103).

\section{Zwei Fallstudien zur ener- gieeffizienten Gebäude- sanierung in Regensburg}

Zwei Fallstudien zur energetischen Sanierung wurden von einem interdisziplinären Team der Ostbayerischen Technischen Hochschule (OTH) Regensburg zusammen mit verschiedenen Stakeholdern initiiert. Zum einen sind hier als wichtige Partner jene zwei Wohnungsbaugenossenschaften zu nennen, die die Sanierungsobjekte besitzen und die Sanierungsmaßnahmen durchführen. Beim Projekt MAGGIE sind eine Reihe weiterer Beteiligter zu nennen, insbesondere die Stadt Regensburg, die Energieagentur Regensburg e. V., ein lokaler Energieversorger und Netzbetreiber sowie Unternehmen aus dem Bau- und Energiegewerbe und Dienstleistungsanbieter.

\section{Beschreibung der Projekte}

In beiden Fällen werden energetische Modernisierungskonzepte entwickelt, die auf regenerative Energieversorgung setzen. Dabei soll der Ensemble-Charakter der historischen Stadtquartiere erhalten bleiben. Die Gesamtwohnkosten sollen durch die Sanierungskosten nicht steigen. Gelingen soll dies durch geringere Energiekosten, die den genossenschaftlich vereinbarten Anstieg der Miete kompensieren. Im Projektverbund RENARHIS ${ }^{1}$ (Dezentrale regenerative Energieversorgung als Schwerpunkt einer nachhaltigen Restaurierung historisch bedeutender Stadtquartiere des frühen 20. Jahrhunderts) wurde exemplarisch für das Plato-Wild-Ensemble im Regensburger Osten aus den 1920er-Jahren von 2014 bis 2016 ein nachhaltiges Sanierungskonzept erarbeitet (Abb. 1).

Das MAGGIE-Projekt ${ }^{2}$ (Energetische Modernisierung des genossenschaftlichen Wohnquartiers Margaretenau in Regensburg) entwickelt und testet von 2017 bis 2021 unter vielfältiger

1 Projektleitung: ОТH Regensburg, Prof. Dr. Oliver Steffens, gefördert durch die Forschungsinitiative „Zukunft Bau“ des Bundesinstituts für Bau-, Stadt- und Raumforschung (Steffens et al. 2016).

2 Projektleitung: OTH Regensburg, Prof. Dr. Oliver Steffens, gefördert vom Bundesministerium für Wirtschaft und Energie (BMWi). Beteiligt sind neben der OTH Regensburg und der Baugenossenschaft Margaretenau die Univer-

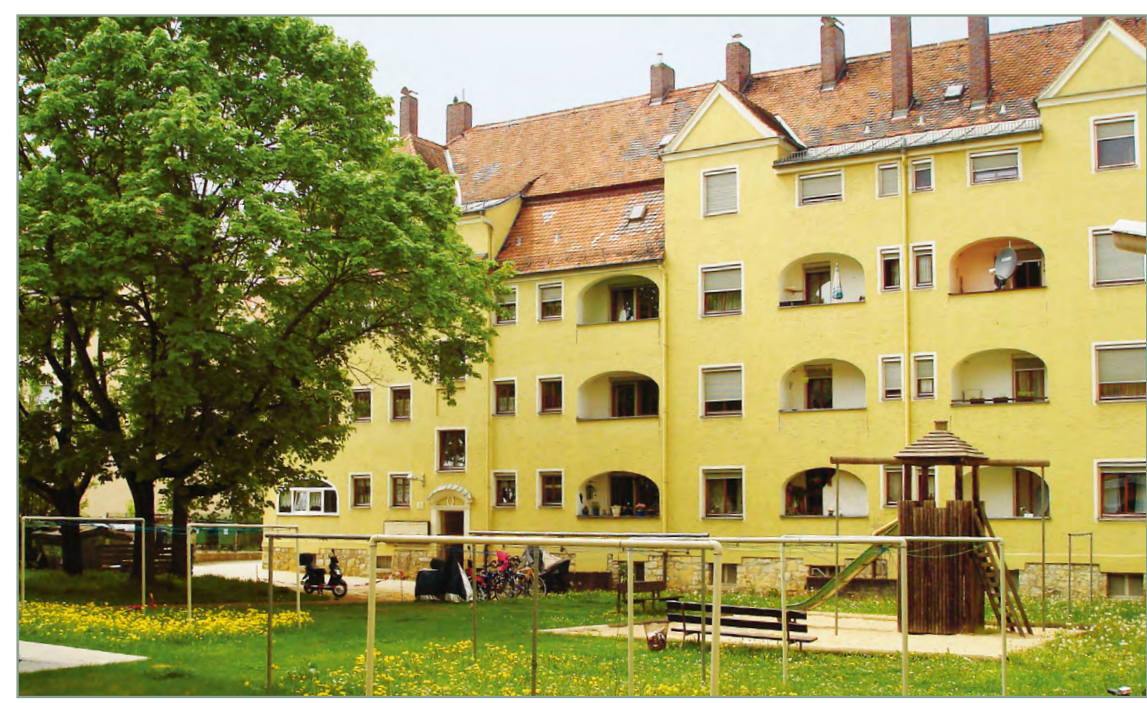

Abb.1: Projekt RENARHIS: Gebäude im Plato-Wild-Ensemble Regensburg.

Quelle: Matthias Vernim, OTH Regensburg

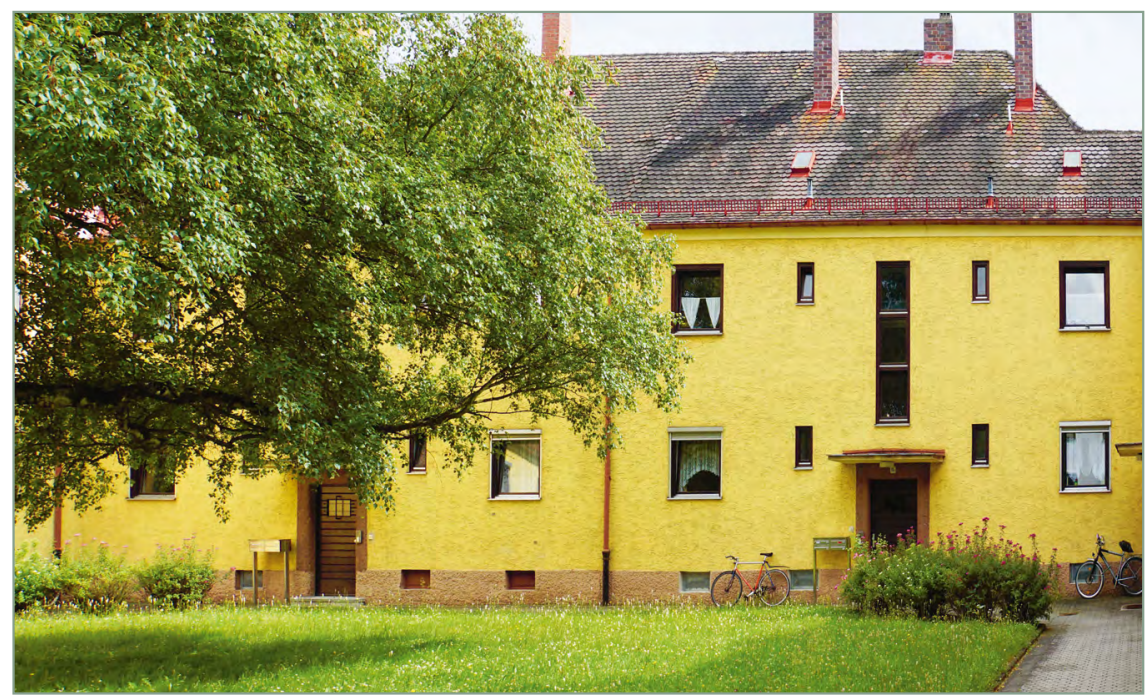

Quelle: Annika Zeitler, ОTH Regensburg

Beteiligung neue Technologien für ein regeneratives, intelligent gesteuertes Energiesystem mit Photovoltaik und Blockheizkraftwerk, solaraktivem Putz und weiteren Maßnahmen an einem Testgebäude der Baugenossenschaft Margaretenau (Abb. 2). Das MAGGIE-Team ist teilweise in das Sanierungsmanagement $(\mathrm{SAMM})^{3}$ involviert. Basierend auf dem MAGGIE-Test wird 2018 bis 2021 für das gesamte baugenossenschaftliche Quartier ein Sanierungskonzept erarbeitet.

sität Bayreuth, die Stadt Regensburg (Amt für Stadtentwicklung), die Energieagentur Regensburg e. V., Franken Maxit Mauermörtel GmbH \& Co., Carnotherm Wärmelogistik GmbH \& Co, Luxgreen GbR, TGA Projektierung GmbH, Kugler + Kerschbaum Partnerschaft, REWAG Regensburger Energie- und Wasserversorgung AG \& Co KG und Consolinno Energy. 
Bei beiden Projekten wurden für eine Sozialstudie Informationen zur soziodemografischen und sozioökonomischen $\mathrm{Zu}$ sammensetzung der Quartiersbevölkerung sowie zur aktuellen Situation, zum zukünftigen Bedarf und zur Akzeptanz der energetischen Sanierung erhoben. Übergeordnetes Ziel ist, die Lebensqualität von Genossenschaftsmitgliedern nachhaltig zu verbessern und die Sanierungsmaßnahmen so sozialverträglich wie möglich zu planen (Haug et al. 2017, S. 580).

\section{Methodik und Durchführung der sozialwissenschaft- lichen Fallstudien}

Durchgeführt wurden die zwei sozialwissenschaftlichen Fallstudien (kurz Sozialstudien) vom Institut für Sozialforschung und Technikfolgenabschätzung (IST) an der OTH Regensburg (Haug et al. 2017, S. 580). Im Plato-Wild-Ensemble des Genossenschaftlichen Bauvereins Regensburg fand im Vorfeld einer geplanten Sanierungsbaumaßnahme und begleitend zur Erstellung eines technischen Gebäudesanierungskonzepts die erste Fallstudie im Projekt RENARHIS statt (Haug und Vernim 2016; Haug und Steffens 2019). Von 158 Wohneinheiten konnten im Befragungszeitraum 14.10.-30.11.2013 in 73 Haushalten teilstandardisierte mündliche Interviews durchgeführt werden (Ausschöpfungsquote $46 \%$ ).

Beim Projekt MAGGIE fand im genossenschaftlichen Quartier Margaretenau im Zeitraum 10.01.-15.02.2019 im Planungsstadium der energetischen Sanierung SAMM eine schriftliche Haushaltsbefragung statt. Der Rücklauf der Vollerhebung betrug 195 von 344 Fragebögen (Ausschöpfungsquote 57\%). Weitere Beteiligungsformen bei MAGGIE waren Informationsbriefe an alle Haushalte, eine Informationstafel im Quartier, eine Infor-
Projekt RENARHIS (Haug und Vernim 2016, S. 54 ff.) sowie Margaretentau für das Projekt MAGGIE. Ausgewertet wurden Alters- und Geschlechtsstruktur, natürliche Bevölkerungsbewegung, Wanderungen, Familienstand, Familienform, Haushaltsgröße, Staatsangehörigkeit sowie Arbeitslosen- und Beschäftigungsquoten. Die Nettomiete liegt in der Margaretenau mit durchschnittlich $5,16 € / \mathrm{m}^{2}$ deutlich unter dem Regensburger Durchschnitt $\left(8,96 € / \mathrm{m}^{2}\right)$.

\section{Ergebnisse}

\section{Sozialstrukturanalyse und Zufriedenheit}

Die Sozialstrukturanalyse zeigt die Besonderheiten in den Wohnbezirken der Safferlingstraße und der Margaretenau auf. Generell ist die Bevölkerung in Regensburg (Stand 31. 12.2017: 166.467 Einwohner) relativ jung und stark durch die Altersgruppe 20 bis 30 geprägt.

Die Bewohnerschaft im Wohnbezirk Safferlingstraße (RENARHIS) war zum Untersuchungszeitpunkt (Stand 31. 12. 2012) etwas jünger als die Regensburger Gesamtbevölkerung und besonders durch Menschen mit Migrationshintergrund geprägt (Haug et al. 2017, S. 591-592). Hinweise auf die prekäre ökonomische Situation der Haushalte geben das relativ niedrige durchschnittliche Pro-Kopf-Einkommen, die hohe Arbeitslosendichte und auch hohe Zahl an Räumungsklagen aufgrund bestehender Mietrückstände (Haug und Vernim 2016, S. 57).

Im Wohnbezirk Margaretenau (MAGGIE) zeigt sich hingegen ein überproportional hoher Anteil an Haushalten mit über 65-Jährigen (Stand 31. 12. 2017), jüngere Personen sind im Ver-

\section{Übergeordnetes Ziel ist, die Lebensqualität von Genossenschaftsmitgliedern nachhaltig zu verbessern.}

mationsveranstaltung mit wissenschaftlichen Vorträgen, eine Begegnungsveranstaltung und Baustellenbesichtigungen. Zur Vertiefung wurden im November 2019 qualitative Interviews mit acht Haushalten aus dem Testgebäude geführt, in dem technische Entwicklungen zur Energiezentrale und Fassadendämmung erprobt wurden. Die Haushalte waren während der Baumaßnahme und Befragung teilweise in Ersatzwohnungen untergebracht.

Bei beiden Projekten wurden zusätzlich aggregierte amtliche Daten der Stadt Regensburg ausgewertet. Die Sozialstrukturanalyse sollte einen Überblick über die soziodemographische Lage der amtlichen Wohnbezirke liefern - Safferlingstraße für das

3 Projektleitung: Tobias Saller, Luxgreen Climadesign, gefördert von der Kreditanstalt für Wiederaufbau (KfW-Bank) im Programm „Energetische Stadtsanierung“ und der Stadt Regensburg (2018). gleich zur Stadt Regensburg sehr viel seltener vertreten. Auch leben dort mehr Ein- oder Zweipersonenhaushalte als Mehrpersonenhaushalte und die Erwerbstätigenquote ist höher als in der Gesamtstadt (Stadt Regensburg, Amt für Stadtentwicklung 2019).

Beide Quartiere verfügen über einen mehr als 100-jährigen Baubestand und weisen Einschränkungen bei der Wohnqualität auf. Insbesondere Probleme mit den Fenstern, der Heizung und Schimmelbildung wurden von den Befragten genannt (Haug und Vernim 2016, S. 71). In der Margaretenau, deren erste Gebäude 1918 errichtet wurden (Baugenossenschaft Margaretenau 2018), berichteten die Haushalte vor allem von störendem Trittschall $(37,5 \%)$ und renovierungsbedürftigen Bädern (25,1\%). Weitere Problembereiche betreffen die Energieversorgung (Heizung und Warmwasser), Schimmelbildung und Müllentsorgung. 
Trotzdem sind die Zufriedenheitswerte der Bewohnenden mit der Wohnungsausstattung und insbesondere mit den Mietkosten hoch (Abb. 3), was sich u. a. durch die geringen Kaltmieten in der Margaretenau erklären lässt, die $45 \%$ unter dem städtischen Durchschnitt liegen (Stadt Regensburg, Amt für Stadtentwicklung 2018, S. 5). Bei Strom- und Heizkosten tritt demgegenüber häufiger Unzufriedenheit auf. Dieser Trend spiegelt sich auch in den Ergebnissen von RENARHIS wider.

\section{Sanierungsmotive}

\section{und Sanierungshemmnisse}

In beiden Befragungen konnte die Bewohnerschaft als offene Antwortmöglichkeiten ihre Hoffnungen und Ängste im Zusammenhang mit der Sanierung formulieren. Die meistgenannte Hoffnung war in beiden Studien mehr Behaglichkeit und Komfort, wobei sie mit $13,3 \%$ in MAGGIE unter dem Wert von RENARHIS mit 16,1\% liegt (Abb. 4). Auf das Einsparen von Heiz- und Energiekosten hoffen verstärkt die Menschen bei MAGGIE, während dies bei RENARHIS mit 3,6\% keine so starke Rolle spielt.

Die meistgeäußerten Befürchtungen stimmen ebenfalls überein. Etwa ein Drittel rechnet mit einer Mieterhöhung (RENARHIS: $36,1 \%$; MAGGIE: $27,7 \%$ ). Ebenfalls befürchtet werden eine lange Sanierungsdauer oder ein notwendiger Auszug.

\section{Akzeptanz des zukünftigen Erneuerbaren-Energien-Systems}

Da beim genossenschaftlichen Wohnbau Mieterinnen und Mieter gleichzeitig stimmberechtigte Mitglieder sind, ist deren Einstellung zu geplanten Maßnahmen entscheidend. Bei RENARHIS wurde als Energiesparmotiv die Umweltschonung mit 59\% fast ebenso häufig wie das Kostensparen mit $61 \%$ genannt. Dem geplanten regenerativen Energiesystem stehen sowohl bei RENARHIS als auch bei MAGGIE viele Haushalte positiv gegenüber (Tab. 1). Bei MAGGIE liegt der Wunsch nach einer Zentralheizung bei 45,2\%, wobei dieser bei Einpersonenhaushalten, bei jüngeren Personen, bei Personen mit geringerer Wohndauer oder bei nicht sehr mietpreissensiblen Personen signifikant stärker ausgeprägt ist als bei der jeweiligen Vergleichsgruppe. In der Margaretenau heizen einige Haushalte noch mit Holz oder Kohle, die über-

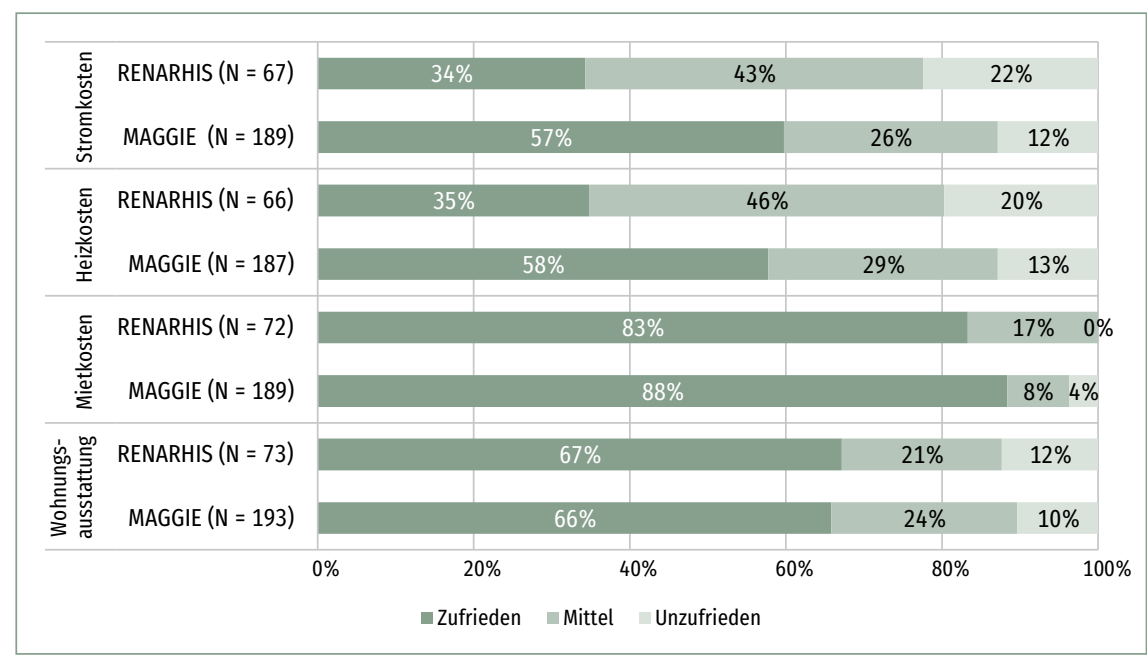

Abb.3: Zufriedenheit mit Strom-, Heiz und Mietkosten sowie Wohnungsausstattung. Quelle: eigene Erhebung RENARHIS (Haug und Vernim 2016, S. 62) und MAGGIE

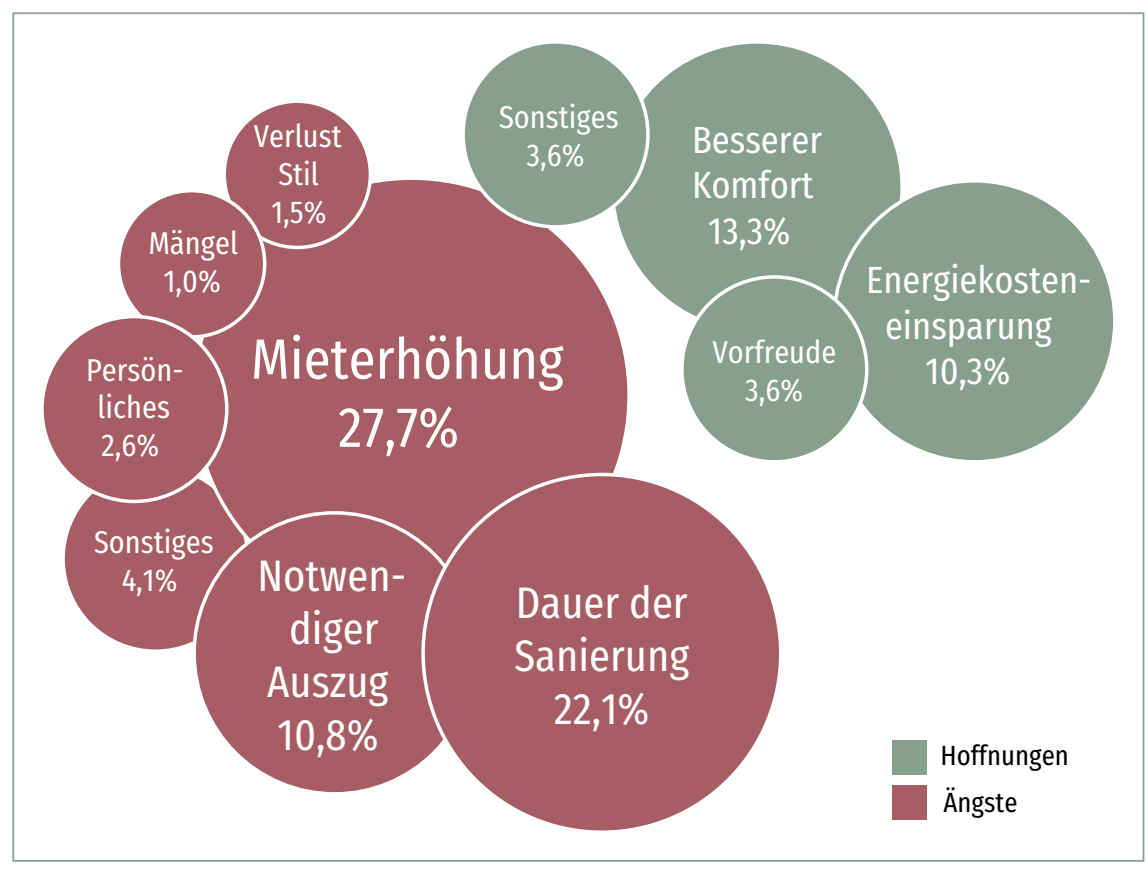

Abb.4: Hoffnungen (grün) und Befürchtungen (rot) bezüglich Sanierungsmaßnahme (offener Nennungen). Quelle: eigene Erhebung MAGGIE Haushaltsbefragung ( $N=195)$

wiegende Mehrzahl jedoch mit Gastherme. Ähnliche Ergebnisse finden sich auch für die zentrale Warmwassererzeugung.

Eine genossenschaftliche Photovoltaikanlage auf dem Dach, die kostengünstig Strom für die Baugenossenschaftsmitglieder erzeugt, erfährt bei MAGGIE sehr hohe Akzeptanz mit 76,1\%. Besonders jüngere Haushalte mit geringer Wohndauer stehen einer genossenschaftlichen Stromversorgung offener gegenüber als andere Personengruppen.

An der deutlichen Zustimmung zu einer Genossenschaftsstromerzeugung ist ein Wunsch nach Selbstversorgung bzw. Au- 
tarkie ablesbar. Genossenschaftlicher Solarstrom entspricht diesem Autarkie-Motiv (Haug et al. 2017, S.602). Der Wunsch nach Selbstbestimmung bzw. Autonomie zeigt sich bei einer Minderheit der befragten Haushalte bei RENARHIS und MAGGIE in einem weniger häufig ausgeprägten Wunsch nach zentraler Warmwasserund Heizenergieerzeugung. (Tab. 1). Ein Teil der Haushalte hat keine Meinung.

\begin{tabular}{|c|c|c|}
\hline Einstellungen zum Energiesystem & RENARHIS & MAGGIE \\
\hline Wunsch Zentralheizung & $44,4 \%$ & $45,2 \%$ \\
\hline Wunsch zentrale Warmwassererzeugung & $45,7 \%$ & $44,2 \%$ \\
\hline Akzeptanz Solarstrom auf dem Dach & $67,6 \%$ & $76,1 \%$ \\
\hline Akzeptanz Mieterhöhung bei Genossenschaftssolarstrom & $65,3 \%$ & $64,7 \%$ \\
\hline N & 73 & 195 \\
\hline
\end{tabular}

Tab.1: Einstellungen gegenüber einem erneuerbaren-Energiesystem und Mieterhöhung.

Quelle: eigene Erhebung RENARHIS und MAGGIE

Bei Interviews in den MAGGIE-Testhaushalten zeigte sich neben einer allgemeinen Technikoffenheit die Durchführung des Forschungsprojekts selbst als akzeptanzförderlich: ,Ja, ich denke, dass es sehr gut ist, weil es ja alles wissenschaftlich ausgewertet wurde schon. Und ich denke, dass das alles eben auf modernsten Stand dann sein soll. Und auch für die Mieter von Vorteil.“

\section{Mieterhöhung, Informationen und Vertrauen in Genossenschaft}

Obgleich der Mietpreis in der Wichtigkeit höher als die Wohnungsausstattung rangiert, zeigte sich bei RENARHIS bereits eine erstaunlich hohe Akzeptanz einer Mieterhöhung bei knapp $70 \%$ im Zusammenhang mit einer neuen Heizung. Vergleichbar würden 65,3\% eine höhere Miete akzeptieren, wenn sie dafür preisgünstigen eigenproduzierten Solarstrom beziehen könnten. Die Ergebnisse von MAGGIE bestätigen mit 64,7\% die Akzeptanz dieses Szenarios (Tab. 1). Gleichzeitig bestehen vielfach Befürchtungen vor einer Kostenbelastung (Abb.4), die sich auch in den Interviews in MAGGIE-Testhaushalten zeigen: „Das andere ist, in wie weit das sozialverträglich ist. Verstehen Sie?“ ,[E]s geht um das Geld und ich kann nicht so weit nach oben gehen.“ „[D]as was wir jetzt hier zahlen, ist ja sowieso echt supergünstig [...]. Von dem her wäre ich da schon einverstanden und würde da ein bisschen mehr zahlen. “Insgesamt wären sechs von acht Haushalten im Testgebäude mit einer Mietkostenerhöhung bis zu maximal $100 €$ einverstanden.

Vertrauen in eine von Genossenschaftsinteressen geleitete Umsetzung spielt ebenfalls eine Rolle bei der Akzeptanz der geplanten Sanierung. Die qualitativen Interviews ergaben, dass Vertrauen teils durch Informationsveranstaltungen aufgebaut wurde, teils bereits zuvor in die Genossenschaft als Institution sowie in ihre handelnden Personen bestand.

\section{Abschließende methodische Reflexion}

Die Befunde sprechen für eine hohe Retest-Reliabilität, da bei der wiederholten Akzeptanzbefragung in zwei Baugenossenschaften annähernd gleiche Ergebnisse erzielt wurden.

Als generelle Schwierigkeit ergibt sich bei der ex-ante-Forschung zur Akzeptanz die Befragung über noch nicht realisierte Sanierungsmaßnahmen (Schäfer und Keppler 2013, S. 76). Das Beschreiben der geplanten technischen Veränderungen scheint oft nur mithilfe komplexer Fachtermini möglich. Einfache Erläuterungen sowie Visualisierungen können jedoch die Ver-

ständlichkeit erhöhen. Bei der ex-ante-Befragung bei RENARHIS und MAGGIE wurden daher Begriffe wie Photovoltaik vermieden und stattdessen nach „Solarstromanlage auf dem Dach“ gefragt. Im MAGGIE-Fragebogen wurden auch Erläuterungen gegeben. So wurde eine Smart-Meter-Anzeige umschrieben mit dem Satz „Es gibt Anlagen, die in der Wohnung eingebaut werden können, an denen abgelesen werden kann, ob gerade Solarstrom erzeugt wird, den Sie günstig nutzen können.". Bei später durchgeführten Interviews im MAGGIE-Testgebäude konnten auch Abbildungen gezeigt werden (z. B. Dämmplatten, Wärmepumpe, Photovoltaikanlage). Eine beantragte ex-post-Befragung zur Technikakzeptanz wurde durch den Fördergeber nicht finanziert.

Zeitdruck, wie er bei Begleitstudien zu energetischer Sanierung häufiger auftritt (ebd., S. 76), erschwerte die Akzeptanzforschung. Ein inhärentes Problem bei zeitlich parallel zur technischen Entwicklung und Testphase verlaufenden Begleitstudien wie MAGGIE ist die Schwierigkeit einer Änderung von Komponenten, die im Förderantrag festgelegt sind. Als vorteilhaft erwies sich hierbei die Verknüpfung des Forschungsprojektes MAGGIE mit dem zeitlich nachgelagerten Sanierungs- und Quartiersmanagement SAMM. So können beim Sanierungsund Quartierskonzept Erkenntnisse zur Realisierbarkeit technischer Innovationen bei der Gebäudesanierung und auch zum Bedarf der Bewohnerinnen und Bewohner an erneuerbarer Energie, barrierearmen Wohnungen, Fahrzeugstellplätzen und nachhaltigen Gemeinschaftseinrichtungen berücksichtigt werden.

\section{Fazit}

Die Ergebnisse zeigen ein hohes Ausmaß an Akzeptanz für die energetische Gebäudesanierung im genossenschaftlichen Wohnbau. Schlussfolgernd werden auf Basis der zwei Fallstudien Akzeptanzfaktoren hervorgehoben. Die im Projekt RENARHIS festgestellten Präferenzmuster und Akzeptanzfaktoren (Haug et al. 2017, S. 602) konnten durch die zweite Fallstudie bestätigt und ergänzt werden.

Partizipation: Aus der hohen Rücklaufquote bei den Haushaltsbefragungen und Teilnahme an Informationsveranstaltungen kann ein Wunsch nach Beteiligung an der Planung der energetischen Modernisierung der genossenschaftlichen Wohnquar- 
tiere abgelesen werden. In genossenschaftlichen Wohnformen bestehen beste Möglichkeiten, partizipative Elemente bei einer energetischen Sanierung einzusetzen. Bei diesen stehen neben der Informationspolitik die Mitbestimmung der Mieterschaft bei der Entscheidung zur Sanierung und das Eingehen auf individuelle Wünsche der Haushalte im Vordergrund.

Vertrauen: Eine bestehende Vertrauensbasis zwischen Bewohnern und Genossenschaftsvorstand ist von hoher Bedeutung. Sind zudem unabhängige Beratungsinstitutionen wie im Fallbeispiel die Energieagentur Regensburg beteiligt, fördert dies zusätzlich, wie Koch und Rosenberger (2019) betonen, das Ver-

\section{Vertrauen in Genossenschaften, Beteiligung und Information erhöht die Akzeptanz von geplanten Sanierungen.}

trauen in die Technik und die beteiligten Dienstleistungsunternehmen. Im Fallbeispiel MAGGIE konnte die ingenieurs- und sozialwissenschaftliche Begleitung zudem als vertrauensförderlicher Faktor wirken. Sofern dieses Vertrauensverhältnis besteht, können Kommunikation und Entscheidung über technische Komponenten an den Vorstand und, im Fallbeispiel MAGGIE, an das Forschungsteam und Sanierungsmanagement delegiert werden.

Sozialverträglichkeit: Ein Sanierungsmotiv ist die Senkung von Energiekosten, wobei Sorgen bzgl. eines Mietkostenanstiegs weit verbreitet sind. Haushalte sind in relativ hohem Maße für eine sozialverträgliche Umsetzung technischer Lösungen offen. Voraussetzung ist, dass durch Effizienzgewinne im Energiebereich ein Anstieg der Gesamtbelastung vermieden wird.

Autarkie: Der Wunsch nach Selbstversorgung ist in der deutlichen Zustimmung zu einer genossenschaftlichen Eigenstromerzeugung bei RENARHIS und MAGGIE ablesbar. Ein Beispiel dafür ist die von vielen Haushalten gewünschte Genossenschaftsstromgewinnung. Während bei der Sanierung des Plato-Wild-Ensembles darauf verzichtet wurde, sollen MAGGIE und SAMM zeigen, wie dies technisch und betriebswirtschaftlich gelingen kann. Bei der aufwändigen Planung ist die Zusammenarbeit verschiedener Stakeholder mit divergierenden Interessen, insbesondere lokaler Stromanbieter und Netzbetreiber, erforderlich.

Als Fazit lässt sich festhalten, dass Baugenossenschaften gute Voraussetzungen mitbringen, um die Sozialverträglichkeit bei einer Gebäudesanierung zu berücksichtigen. Als vorteilhaft erwies sich gemäß den Empfehlungen von Tappeiner et al. (2004) oder Wehnert et al. (2007) ein interdisziplinäres Team unter Einbindung unterschiedlicher Stakeholder. Weiteren Forschungsbedarf gibt es in Bezug auf die Frage der Zufriedenheit nach der Sanierung.

\section{Literatur}

Atmaca, Delal (2014): Genossenschaften in Zeiten raschen Wandels. Chancen einer nachhaltigen Organisationsform. In: Carolin Schröder und Heike Walk (Hg.): Genossenschaften und Klimaschutz. Akteure für zukunftsfähige, solidarische Städte. Wiesbaden: Springer VS, S. 49-72. DOI: 10.1007/978-3-65803632-4_3

Baugenossenschaft Margaretenau e. G. (2018): 100 Jahre Baugenossenschaft Margaretenau 1918-2018. „Es ist eine Gnade hier zu leben“. Regensburg: Friedrich Pustet.

BMVBW - Bundesministerium für Verkehr, Bau und Wohnungswesen (2004): Wohnungsgenossenschaften. Potenziale und Perspektiven. Bericht der Expertenkommission Wohnungsgenossenschaften. Berlin: Duncker \& Humblot.

Böhm, Uta; Schäfer, Martina; Stadler, Maria (2019): Energieeffizienz im Spannungs feld zwischen Anlagentechnik und sozialen Akteuren. Hemmnisse für den effizienten Betrieb komplexer Heizungsanlagen. In: TATuP - Zeitschrift für Technikfolgenabschätzung in Theorie und Praxis 29 (3), S. 55-61. D0I: 10.14512 / tatup.28.3.55.

Deutsche Energie-Agentur (2018): Dena Gebäudereport Kompakt 2018. Statistiken und Analysen zur Energieeffizienz im Gebäudebestand. Online verfügbar unter https://www.dena.de/fileadmin/dena/Dokumente/Pdf/9254_ Gebaeudereport_dena_kompakt_2018.pdf, zuletzt geprüft am 26. 02.2020.

Diekmann, Andreas; Preisendörfer, Peter (2001): Umweltsoziologie. Eine Einführung. Reinbek bei Hamburg: Rowohlt-Taschenbuch-Verlag. DOI: 10.1007/ s11577-001-0123-1.

Gossen, Maike; Nischan, Carolin (2014): Regionale Differenzen in der Wahrnehmung energetischer Sanierungen. Ergebnisse einer qualitativen Befragung von GebäudeeigentümerInnen zu energetischer Sanierung in zwei unterschiedlichen Regionen. Online verfügbar unter https://www.gebaeudeenergiewende.de/data/gebEner/user_upload/Dateien/GEW_AP1_ Ergebnisbericht_Interviews_final_141126.pdf, zuletzt geprüft am 26.02.2020. Hacke, Ulrike; Lohmann, Günter (2006): Akzeptanz energetischer Maßnahmen im Rahmen der nachhaltigen Modernisierung des Wohnungsbestandes. Abschlussbericht. Bundesamt für Bauwesen und Raumordnung. Online verfügbar unter https://www.iwu.de/fileadmin/user_upload/dateien/energie/ klima_altbau/Endbericht_Akzeptanz_energ_Massnahmen.pdf, zuletzt geprüft am 20.11.2019.

Haug, Sonja; Steffens, Oliver (2019): Energetische Gebäudesanierung. Die Akzeptanz von Baumaßnahmen im Planungsstadium. In: Deutsches Ingenieurblatt (3), S. 20-27.

Haug, Sonja; Vernim, Matthias (2016): Sozialstudie. In: Oliver Steffens et al. (Hg.): RENARHIS. Nachhaltige energetische Modernisierung und Restaurierung historischer Stadtquartiere. Zukunft Bauen, Forschung für die Praxis, Band 3. Bonn: Bundesinstitut für Bau- Stadt- und Raumforschung im Bundesamt für Bauwesen und Raumordnung, S. 47-73.

Haug, Sonja; Weber, Karsten; Vernim, Matthias (2017): Soziale und planerische Aspekte der energetischen Gebäudemodernisierung. Partizipative Planung, Zielkonflikte und Akzeptanz. In: Katrin Großmann, André Schaffrin und Christian Smigiel (Hg.): Energie und soziale Ungleichheit. Zur gesellschaftlichen Dimension der Energiewende in Deutschland und Europa. Wiesbaden: Springer VS, S. 579-610. DOI: 10.1007/978-3-658-11723-8_22.

Heinze, Rolf; Bölting, Torsten (2019): Strukturwandel an der Ruhr und die transformative Kraft der Emschergenossenschaft. In: Rolf Heinze, Uli Paetzel und Torsten Bölting (Hg.): Wasser, Wohnen, Werte. Genossenschaften 
stiften Mehrwert. Konferenzbroschüre. Essen: Emschergenossenschaft, S. 24-73.

Kamburow, Christian; Illge, Lydia; Oertel, Britta (2016): Akzeptanzfaktoren für die Energiedienstleistung „Mieterstrom“ am Beispiel der Stadt Essen. In: Marco Koch, Heike Jacobsen und Britta Oertel (Hg.): Wettbewerb „Energieeffiziente Stadt“. Band 7: Dienstleistungen für die energieeffiziente Stadt. Berlin: LIT Verlag, S. 103-110.

Koch, Carmen; Rosenberger, Nicole (2019): Ganzheitliche Betrachtung, Vertrauen und Erfahrungsaustausch. Kommunikation mit Hauseigentümern über energetisches Sanieren. Online verfügbar unter https://digitalcollection.zhaw.ch/ bitstream/11475/15017/1/WorkingPapers\%2017_Koch_Rosenberger.pdf, zuletzt geprüft am 15.10.2019.

Ostrom, Elinor (1999): Die Verfassung der Allmende. Jenseits von Staat und Markt. Tübingen: Mohr Siebeck.

Schäfer, Martina; Keppler, Dorothee (2013): Modelle der technikorientierten Akzeptanzforschung. Überblick und Reflexion am Beispiel eines Forschungsprojektes zur Implementierung innovativer technischer EnergieeffizienzMaßnahmen. Online verfügbar unter http://www.tu-berlin.de/fileadmin/ f27/PDFs/Discussion_Papers/Akzeptanzpaper__end.pdf, zuletzt geprüft am 28.11.2019.

Stadt Regensburg (2018): Sanierungsmanagement Margaretenau. Online verfügbar unter http://www.regensburg-effizient.de/energie-und-klimaschutzin-regensburg/quartierskonzepte/margaretenau/, zuletzt geprüft am 16. 03.2020.

Steffens, Oliver et al. (Hg.) (2016): RENARHIS. Nachhaltige energetische Modernisierung und Restaurierung historischer Stadtquartiere. Zukunft Bauen, Forschung für die Praxis, Band 3. Bonn: Bundesinstitut für Bau- Stadt- und Raumforschung im Bundesamt für Bauwesen und Raumordnung.

Tappeiner, Georg; Walch, Karin; Koblmüller, Manfred; Loibl, Celine; Lukovnjak, Martin (2004): Sanierung Pro! Sanierung und Partizipation im mehrgeschossigen Wohnbau. Wien: Österreichisches Ökologie Institut. Online verfügbar unter https://www.nachhaltigwirtschaften.at/resources/hdz_pdf/endbericht_ sanierung_pro_id2774.pdf, zuletzt geprüft am 18.11.2019.

Vögele, Stefan et al. (2016): Reduktion des gebäuderelevanten Energiebedarfs als Herausforderung für die Energiewende. Sechs Thesen zu unterschätzten Barrieren und Potenzialen. In: Policy Brief. Online verfügbar unter http://www.energy-trans.de/downloads/ENERGY-TRANS-PolicyBrief\%20 W\%C3\%A4rmewende.pdf, zuletzt geprüft am 31. 10.2019.

Wehnert, Timon et al. (2007): Erneuerbare Energien in Kommunen optimal nutzen. Denkanstöße für die Praxis. Berlin: Institut für Zukunftsstudien und Technologiebewertung (IZT). Online verfügbar unter https://www.izt.de/ fileadmin/downloads/pdf/SKEP/SKEP_EE_in_Kommunen.pdf, zuletzt geprüft am 16.07.2020.

Weiß, Julika; Dunkelberg, Elisa; Stieß, Immanuel; Zundel, Stefan (2012): Klimaschutz durch energetische Gebäudesanierung. In: Ökologisches Wirtschaften (1), S. 14-15. Online verfügbar unter https://oekologisches-wirtschaften.de/ index.php/oew/article/viewFile/1174/1171, zuletzt geprüft am 02.07.2020.

Wilson, Charlie; Crane, Lucy; Chryssochoidis, George (2015): Why do homeowners renovate energy efficiently? Contrasting perspectives and implications for policy. In: Energy Research and Social Science (7), S. 12-22. D0I: 10.1016/ j.erss.2015.03.002.

Wüstenhagen, Rolf; Wolsink, Maarten; Bürer, Mary Jean (2007): Social acceptance of renewable energy innovation. An introduction to the concept. In: Energy Policy (35), S. 2683-2691. DOI: 10.1016/j.enpol.2006.12.001.

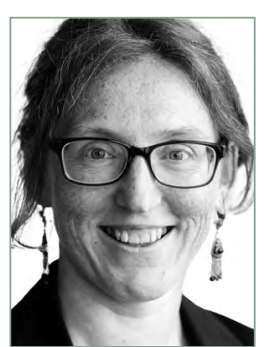

PROF.DR. SONJA HAUG

ist Soziologin und seit 2010 Professorin für Empirische Sozialforschung an der Ostbayerischen Technischen Hochschule (OTH) Regensburg. Gemeinsam mit Prof. Dr. Weber leitet sie das Institut für Sozialforschung und Technikfolgenabschätzung (IST). Ihre inhaltlichen Schwerpunkte liegen einerseits im Bereich Demografie, Familie und Migration, andererseits bei Studien zu sozialen Aspekten von Technik in den Bereichen Energie, Mobilität und Gesundheit. Sie leitete die Sozialstudien zu RENARHIS und MAGGIE.

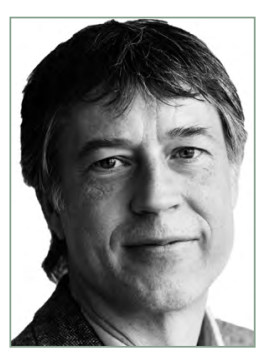

\section{PROF.DR. KARSTEN WEBER}

ist Ko-Leiter des Instituts für Sozialforschung und Technikfolgenabschätzung (IST) der OTH Regensburg, einer der drei Direktoren des Regensburg Center of Health Sciences and Technology (RCHST) sowie Honorarprofessor für Kultur und Technik an der BTU Cottbus-Senftenberg. Er beschäftigt sich derzeit mit individuellen und gesellschaftlichen Auswirkungen von luk-Technologie sowie mit wertebasierter Gestaltung von Technik insbesondere im Gesundheitsbereich.

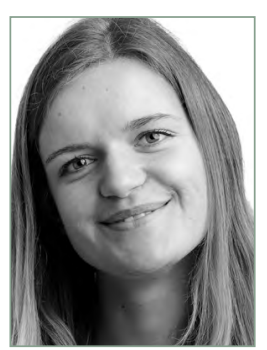

\section{MIRIAM VETTER}

hat Soziale Arbeit an der OTH Regensburg studiert. Im Anschluss absolvierte sie den Master der Sozialen Arbeit mit dem Schwerpunkt Inklusion und Exklusion. Seit 2019 ist sie wissenschaftliche Mitarbeiterin im Institut für Sozialforschung und Technikfolgenabschätzung (IST) an der OTH Regensburg. Sie bearbeitet die Sozialstudie, welche das MAGGIEProjekt aus sozialwissenschaftlicher Perspektive begleitet. Ihre Schwerpunkte sind soziale Aspekte der Gebäudesanierung und Altersforschung. 